Tropical Journal of Pharmaceutical Research October 2020; 19 (10): 2153-2159

ISSN: $1596-5996$ (print); 1596-9827 (electronic) (C) Pharmacotherapy Group, Faculty of Pharmacy, University of Benin, Benin City, 300001 Nigeria

\title{
The mushroom, Cordyceps cicadae, ameliorates renal interstitial fibrosis via TLR2-mediated pathways
}

\author{
Rong Zheng, Yuhuang Wang, Yifei Zhong*, Rong Zhu \\ Department of Nephrology, Longhua Hospital, Shanghai University of Traditional Chinese Medicine, 725 Southern Wanping \\ Road, Shanghai 200032, China \\ *For correspondence: Email: Ihkidney_zr@126.com; yifeilily@126.com \\ Sent for review: 25 February 2020 \\ Revised accepted: 20 September 2020
}

\begin{abstract}
Purpose: To evaluate the mushroom, Cordyceps cicadae, for its ability to suppress tissue fibrosis and Toll-like receptors 2 (TLR 2) pathway activation in a mouse model of renal interstitial fibrosis (RIF). Methods: Cordyceps cicadae powder was obtained from BioAsia Group (Shanghai, China). RIF was induced via unilateral ureteral obstruction (UUO) in male C57BI/6 mice. Animals were treated via the intragastric administration of Cordyceps cicadae powder $(0.1 \mathrm{~g}, 0.3 \mathrm{~g} / \mathrm{ml} / 100 \mathrm{~g} /$ day), beginning $24 \mathrm{~h}$ prior to UUO, and the treatment was continued for the following 14 days. Changes in tissue histology were then assessed via hematoxylin and eosin, and Sirius red stainings. Tissue macrophages were characterized based upon their expression of inducible nitric oxide synthase (iNOS) and interleukin-10 (IL-10), while Western blotting technique was used to measure the levels of TLR2, Myeloid differentiation factor 88 (MyD88), and nuclear factor-KB (NF-KB)/p-NF-KB in samples from these animals.

Results: Treatment with Cordyceps cicadae powder is associated with a shift in macrophage phenotype that in turn decreased the production of extracellular matrix and alleviated RIF occurrence in mice model.

Conclusion: This mechanistic study highlights the novel potential approach for treating and preventing RIF using Cordyceps cicadae powder.
\end{abstract}

Keywords: Renal interstitial fibrosis, TLR2-mediated pathway, Cordyceps cicadae

\begin{abstract}
This is an Open Access article that uses a funding model which does not charge readers or their institutions for access and distributed under the terms of the Creative Commons Attribution License (http://creativecommons.org/licenses/by/4.0) and the Budapest Open Access Initiative (http://www.budapestopenaccessinitiative.org/read), which permit unrestricted use, distribution, and reproduction in any medium, provided the original work is properly credited.
\end{abstract}

Tropical Journal of Pharmaceutical Research is indexed by Science Citation Index (SciSearch), Scopus, International Pharmaceutical Abstract, Chemical Abstracts, Embase, Index Copernicus, EBSCO, African Index Medicus, JournalSeek, Journal Citation Reports/Science Edition, Directory of Open Access Journals (DOAJ), African Journal Online, Bioline International, Open-J-Gate and Pharmacy Abstracts

\section{INTRODUCTION}

Cordyceps cicadae (C. cicadae), a mushroom having medicinal values, primarily grows in the subtropical regions of China and derives its nutrient contents from Cicada flammata Dist. larvae. C. cicadae has been employed in the preparation of Chinese herbal medicines for millennia and is an entomogenous fungus of the
Cordyceps genus of ascomycete fungi, which is composed of roughly 400 other described species [1]. Historically, C. cicadae has been utilized to treat asthma and convulsions [2]. In recent years, it has been used to preserve renal functions [3,4], and treat cancer. Besides, it has also been reported that it can effectively combat helminthiasis and inhibit acetylcholinesterase activity [5]. We have previously demonstrated 
that C. cicadae extracts are capable of ameliorating renal fibrosis in vivo and in vitro. However, the mechanistic basis for such protective activity has not been elucidated to date.

Chronic kidney disease (CKD) is a heterogeneous series of disorders that ultimately drive renal functional and structural decline over a period of months or years, thus causing significant morbidity and mortality in affected individuals [6,7]. Currently, treatment or prevention of end-stage renal disease (ESRD) has been the focus of an active pharmacological investigation. In contrast to many modern pharmaceuticals that have been recently designed, many traditional Chinese medicines (TCMs) have been utilized as renoprotective or diuretic agents for centuries, and are welldocumented in TCM pharmacopeias [8]. These TCM preparations have rarely been studied in well-designed preclinical or clinical trials, thus their therapeutic applications remain relatively limited. It is, therefore, essential to conduct comprehensive and rigorous analyses for TCMs for gauging their therapeutic efficacy appropriately. This is particularly important for rational selection of appropriate TCMs for improving therapeutic outcomes in CKD patients. The present research work was designed to examine the mushroom $C$. cicadae for its tissue fibrosis suppressing ability in an animal model of renal interstitial fibrosis (RIF).

\section{EXPERIMENTAL}

\section{Drug and animals}

Cordyceps cicadae powder was obtained from BioAsia Group (Shanghai, China). C. cicadae strain was isolated and purified from the natural C. cicadae collected in Guniujiang nature reserve of Anhui Province by BioAsia Group and was selected by artificial breeding. The natural specimens were preserved in the specimen room of BioAsia Group. Further, to preserve and maintain the microbial samples, the strains were deposited in the CGMCC (China Microbiological Culture Collection Center) with specimen no. CGMCC 6396. According to the morphological characteristics of Institute of Microbiology, the Chinese Academy of Sciences, and ITS gene sequence comparison in the NCBI database, the identified $C$. cicadae strain was inoculated on artificial medium, and the fruiting body was obtained by liquid-solid two-phase fermentation. After harvesting, the pure powder was made through modern ultra-fine grinding technology.
Male C57BL6/J mice (20-25g) were received from the Shanghai SLAC Laboratory Animal Center (China) and were housed under standard conditions in a animal facility at $22 \pm 1{ }^{\circ} \mathrm{C}$ with a $12 \mathrm{~h}$ light/dark cycle. A unilateral ureter obstruction (UUO) model was used to simulate CKD and renal interstitial fibrosis (RIF) in mice. Briefly, animals were allowed to acclimatize to the housing facility for one week, after which they were randomly allocated into four groups ( $\mathrm{n}=4$ /group): a sham group $\left(\mathrm{dH}_{2} \mathrm{O} \quad 1 \mathrm{ml} / 100\right.$ g/day), a UUO group (UUO mice treated with $\mathrm{dH}_{2} \mathrm{O} 1 \mathrm{ml} / 100 \mathrm{~g} / \mathrm{d}$ ), a UUO + low-dose treatment group (UUO mice treated with $C$. cicadae treatment $0.1 \mathrm{~g} / \mathrm{ml} / 100 \mathrm{~g} / \mathrm{d}$ ), and a UUO + highdose treatment groups (UUO mice treated with C. cicadae $0.3 \mathrm{~g} / \mathrm{ml} / 100 \mathrm{~g} /$ day).

Dosing to the animals was initiated via oral gavage $24 \mathrm{~h}$ prior to UUO or sham modeling and was sustained for 14 days after modeling. UUO modeling was conducted as reported in prior studies [9]. Briefly, animals were anesthetized using inhaled ether, after which the left ureter was located via a left lateral incision and was subjected to 3-0 wire double ligation. Shamoperated animals underwent identical surgical procedures, but ureter ligation was not performed. At 14 days post-UUO, mice were euthanized and kidney samples were collected and stored at $-80{ }^{\circ} \mathrm{C}$ for histological evaluation and Western blotting. The Institutional Animal Care and Use Committee of Longhua Hospital, Shanghai University of Traditional Chinese Medicine, Shanghai, PR China, gave approval for the study. The study was carried out in line with the "Principles of Laboratory Animal Care" (NIH 85-23, 1985) [10].

\section{Histopathological examination}

Hematoxylin and eosin (HE) staining of renal tissue sections from the animals was used to gauge cellular infiltration, general tissue histology, and extracellular matrix deposition in the kidneys of UUO model animals. These sections were evaluated by using the semiquantitative renal tubulointerstitial injury index based upon the degree of interstitial fibrosis, tubular atrophy, and interstitial fibrosis as follows: Grade $0=$ normal, with no fibrosis, no changes in tubular morphology, and minimal evidence of inflammatory cell infiltration; Grade $1=$ slight evidence of atrophy, with focal necrosis or degeneration of the renal tubular epithelial cells, scattered inflammatory cell infiltration, and mild fibrotic hyperplasia with lesions accounting for < $25 \%$ of total tissue area; Grade 2 = moderate tissue atrophy, with more widespread necrosis or degeneration of the renal tubular epithelial cells, 
mild inflammatory cell infiltration, and moderate fibrotic hyperplasia with lesions accounting for 26 - $50 \%$ of total tissue area; Grade 3 = substantial atrophy, with clear renal tubular epithelial cell necrosis and degeneration, substantial diffuse or clustered inflammatory cell infiltration, and bundles of fibrotic tissue with lesions accounting for $>50 \%$ of total tissue area. These grades were respectively assigned scores of $0,1,2$, and 3 points. A total of 10 random fields of view per tissue section (400x) were evaluated and scored; with the average score for each sample, then the final renal tubulointerstitial injury score was calculated for each specimen.

Masson's trichrome staining was used to detect the collagen deposition (stained in blue) in the renal tissue of studied animals. A total of 10 random fields of view per stained tissue section (200x) were evaluated to calculate the fibrotic area as a fraction of total interstitial area (excluding the glomeruli, tubules, and blood vessels), using a high-definition color pathological image and text analysis system.

Sirius Red staining was also utilized to detect the collagen deposition (stained in red) in kidney tissue sections obtained from the experimental mice, with interstitial dilatation. A total of 10 random fields of view (200x) per section were analyzed for type I and type III collagen deposition and were analyzed with a highdefinition color pathological image analysis system.

\section{Western blotting}

Tissue samples were lysed using a buffer supplemented with protease inhibitors (Merck). A BCA kit (Pierce) was then used to quantify protein levels in these lysates, which were subsequently separated via $12 \%$ SDS-PAGE and transferred to nitrocellulose membranes. Blots were then blocked for $30 \mathrm{~min}$ with $5 \%$ non-fat milk in TBST, followed by overnight incubation with anti- transforming growth factor- $\beta 1$ (TGF- $\beta$ 1) (Santa Cruz Biotechnology), anti- interleukin10 (IL-10)(Abcam), anti- nuclear factor-kB (NFkB)(Abcam), anti-p-NF-kB (Abcam), anti- myeloid differentiation factor 88 (MyD88)(Santa Cruz Biotechnology), Anti- Toll-like receptors 2 (TLR 2) (Santa Cruz Biotechnology), or anti- inducible nitric oxide synthase (iNOS) (Santa Cruz Biotechnology).

\section{Statistical analysis}

SPSS 17.0 software (SPSS, IL, USA) was used for all statistical testing in this study. Data are given as means \pm Standard Error of Mean (SEM) and were compared via one-way analyses of variance (ANOVAs), with $\mathrm{P}<0.05$ as the significance threshold.

\section{RESULTS}

\section{C. cicadae administration ameliorates renal tissue injury in UUO model mice}

In order to compare difference in the severity of renal tissue injury between UUO model animals, treated with and without C. cicadae, ligated and contralateral kidneys in the animals were assessed at two weeks post-surgery. To evaluate any potential compensatory changes observed in the contralateral kidney, animals in the sham group served as controls. Findings of the study revealed that UUO treatment resulted in hydronephrotic kidney enlargement, while $C$. cicadae treatment alleviated this symptom in a dose-dependent manner.

H\&E-stained renal sections from the experimental mice indicated that $C$. cicadae treatment significantly reduced kidney tissue damage. Furthermore, the renal tubulointerstitial injury scores revealed similar conclusion (Figure 1). Masson's trichrome staining revealed that renal tissue samples from UUO model group animals exhibited a significantly larger fibrotic area relative to that observed in tissues from sham-operated controls $(p<0.05)$. Importantly, C. cicadae treatment alleviated this RIF in a dose-dependent fashion $(p<0.01)$. Similarly, Sirius Red staining indicated that treatment with C. cicadae was sufficient to reduce collagen deposition in the ligated kidneys of UUO mice (Figure 1). On the other hand, animals in UUO model group exhibited significantly higher levels of interstitial type I and type III collagen relative to sham controls $(P<0.01)$, these levels were considerably lower in $C$. cicadae-treated animals relative to those in model group animals $(P<$ $0.01)$. This suggests that $C$. cicadae was able to relieve hydronephrosis and ameliorate renal injury in UUO-induced model of murine RIF.

\section{Impact of C. cicadae treatment on macrophage phenotypes in UUO mice}

Macrophages are key regulators of RIF development and progression. They can undergo context-dependent differentiation in response to local microenvironmental changes, thus lead these cells to adopt a pro-inflammatory (M1) or anti-inflammatory (M2) phenotype. Similarly, these macrophages can not only facilitate tissue repair in certain contexts but can also promote pathological tissue fibrosis in other settings. As such, the M1/M2 ratio can be used to gauge the 

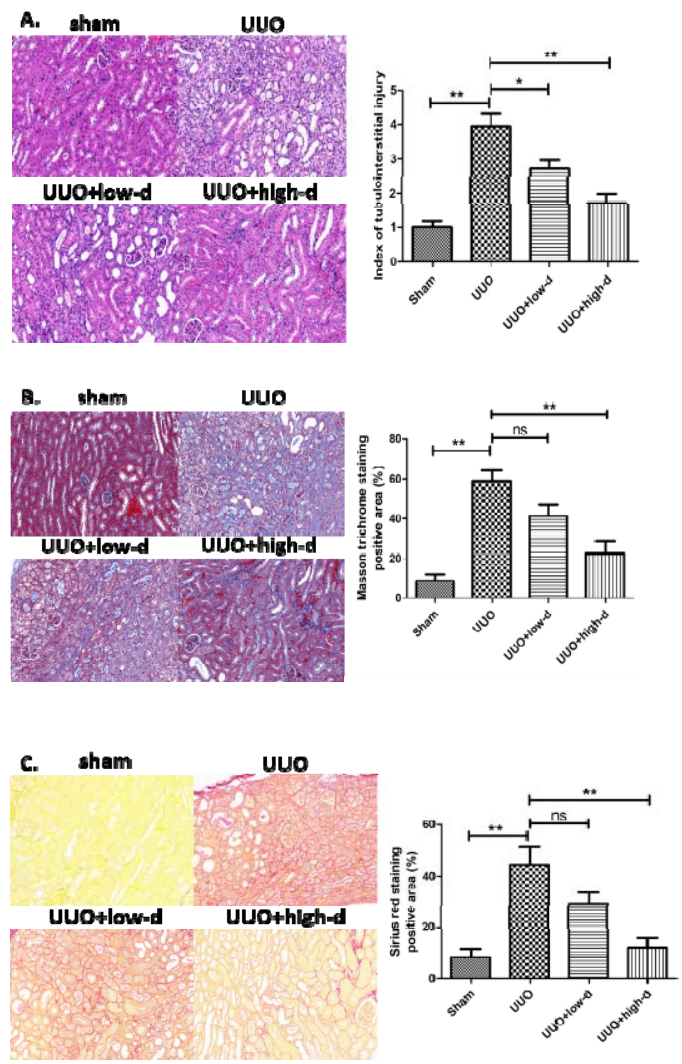

Figure 1: The impact of Cordyceps cicadae on renal tissue in UUO model mice. (A) H\&E staining demonstrated that $C$. cicadae treatment reversed UUO-mediated impairment of renal parenchymal cell integrity in a dose-dependent fashion. Stained slides were scored in a semi-quantitative fashion based on the fibrotic tissue area using the following scale: 0 , none; 0.5 , < $10 \% ; 1,10-25 \% ; 2,25-50 \%$; 3, $50-$ $70 \%$; and 4, >75 \%. (B) Masson's trichrome staining revealed substantial collagen deposition (blue) in ligated kidneys from UUO model animals, whereas $C$. cicadae treatment reduced this collagen deposition. (C) Sirius Red staining demonstrated that UUO resulted in significant increases in interstitial collagen deposition (red) that were reduced in response to $C$. cicadae treatment. Magnification: 200x. ImageJ was used for quantitative analyses of connective tissue area. Data are mean \pm SEM; ${ }^{*} p<0.05$ and ${ }^{* *} p<0.01$. Scale bar $=100 \mathrm{uM}$

relative fibrotic and inflammatory activity of macrophages within the renal tissue samples. To that end, we employed Western blotting to measure the expression of the $M 1$ and $M 2$ markers iNOS and IL-10, respectively, in renal tissue samples from our UUO model mice. We found that iNOS levels were the highest in untreated UUO model animals and that these levels fell in a dose-dependent manner in response to $C$. cicadae treatment. On the other hand, IL-10 levels increased in these treated animals (Figure 2). These results suggest that $C$. cicadae was able to disrupt M1 macrophage infiltration of the kidneys, instead of driving M2 macrophage accumulation, activation, and consequent IL-10 production. Given its welldocumented anti-inflammatory roles, IL-10 may in turn be a primary mechanism whereby $C$. cicadae can help to normalize renal tissue function in treated mice.

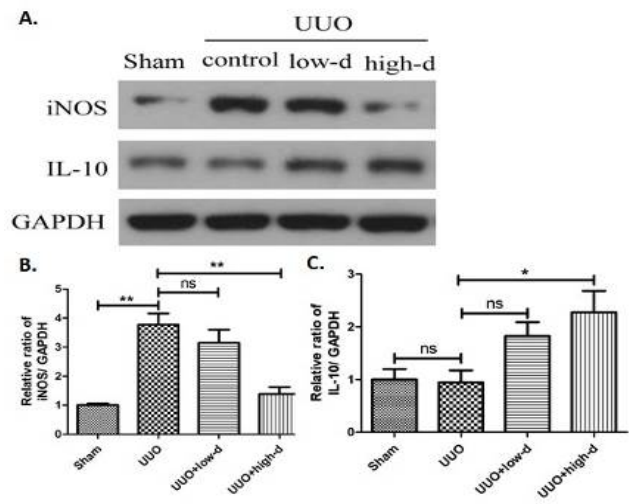

Figure 2: Impact of C. cicadae treatment on macrophage phenotypes in UUO model mice. Western blotting was carried out to examine the levels of M1 and M2 macrophages in renal tissue samples based on the relative levels of inducible nitric oxide synthase (iNOS) and IL-10, respectively (A), with relative protein levels being normalized to GAPDH levels in a semiquantitative fashion $(B, C)$. Data are means $\pm S E M ;{ }^{*} p$ $<0.05$ and ${ }^{* *} p<0.01$

\section{C. cicadae inhibits TLR2 signaling}

In order to assess the ability of $C$. cicadae treatment for modulating TLR2 signaling in treated mice, we next evaluated the expression of proteins associated with the TLR2 signaling pathway MyD-88, and NF-kB, p-NF-kB via Western blotting (Figure 3 ). We found that UUO was associated with significant increases in renal TLR2 and MyD88 levels, while $C$. cicadae treatment reduced TLR2, MyD88, and p-NF-kB levels in a dose-dependent fashion after UUO.

\section{DISCUSSION}

RIF can be induced by damage arising as a consequence of renal injury or pathogen infection. The integrity and functionality of renal tissues are further compromised by the inappropriately regulated tissue repair responses. RIF development and progression is mediated by inflammation, the apoptotic death of renal cells, oxidative stress, and the excessive production of pro-fibrotic cytokines $[11,12]$. Here in the existing study, we examined the potential of C. cicadae in the alleviation of the RIF development using an animal model. 


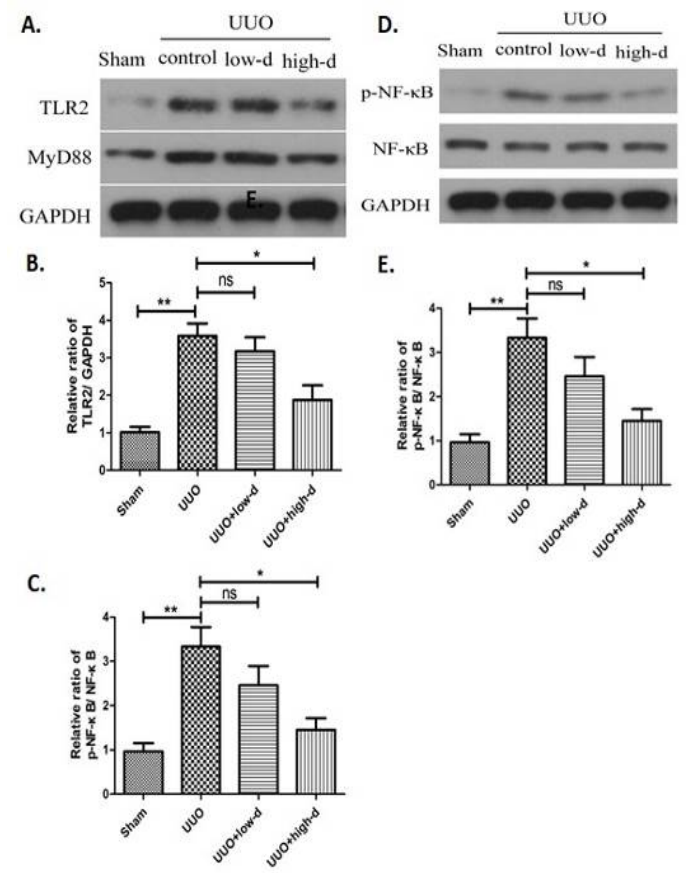

Figure 3: The impact of $C$. cicadae treatment on TLR2 signaling pathways. (A) TLR2 and MyD88 levels in renal tissue samples were assessed via Western blotting, (B-C) and were normalized to GAPDH in order to assess relative protein expression in a semiquantitative fashion. (D) Levels of $\mathrm{p}-\mathrm{NF}-\mathrm{kB}, \mathrm{NF}-\mathrm{kB}$, and GAPDH were assessed in renal tissue samples via Western blotting, (E) and the ratio of $p-N F-k B$ to NF-KB in these blots was calculated. Data are means \pm SEM. ${ }^{*} P<0.05$ and ${ }^{* *} P<0.01$

We found that $C$. cicadae oral administration significantly lowered the fibrosis related protein aggregates and restored the ratio of $\mathrm{M} 1 / \mathrm{M} 2$ macrophages, which related to the TLR2/MyD88 and signaling pathway of NF-KB in the ligated kidney.

We have previously demonstrated that $C$. cicadae treatment reduced serum creatinine (Scr) and blood urea nitrogen (BUN), while simultaneously increasing the endogenous creatinine clearance rate (GFR), plasma albumin (ALB), and hemoglobin (HB) levels and reducing urinary protein levels in preclinical models $[3,4,13]$. At a mechanistic level, we found that the ability of $C$. cicadae to treat CKD was associated with the suppression of epithelial-mesenchymal transition and inflammation. Growing evidences show that it is related to the polarity of macrophages. M1 macrophages serve as key drivers of tissue fibrosis and are largely induced in response to stimuli including lipopolysaccharide (LPS) and interferon-y (IFNY), whereupon these activated cells can secrete inflammatory factors i.e., tumor necrosis factor- $\alpha$ (TNF- $\alpha$ ), IL-1, IL-6, and IL-12. M1 macrophages further produce nitric oxide (NO) and reactive oxygen species (ROS) that cause proximal tissue injury, and they secrete macrophage chemotactic protein-1 (MCP-1) and other chemokines including CCL2, CCL3, CCL4, and CCL20/macrophage inflammatory protein 3a (MIP-3a), which in turn recruit other damaging inflammatory cells to the site of tissue injury. M1 macrophages are the predominant cell type observed during early stages of tissue injury, and eliminating these cells might be a viable approach to alleviating the long-term development of tissue fibrosis [14].

The pathogenic nature of macrophages in the context of RIF has been validated by depletion experiments wherein the elimination of macrophages using specific antibodies or clodronate liposomes was enough to alleviate inflammation and tissue fibrosis in a model of experimental acute renal injury [15]. Thus, disrupting M1 macrophage activation in the context of RIF in vivo represents an attractive therapeutic approach that may offer a novel means of preventing or treating tissue fibrosis [16-18]. In our study, we analyzed the levels of M1 macrophages via iNOS. Here, we have examined a decreased expression level of iNOS in the result of renal ligation; which suggests a decrease in M1 activity. These results suggest that $C$. cicadae may have an effective role in intiinflammation and disrupting M1 macrophage activation. However, there is a need for extensive study to evaluate the underlined phenomenon of macrophages.

TLRs are the key mediators of innate immune system activation and serve to bridge the gap between the innate and adaptive immune system. TLR activation is also closely associated with the development of RIF [19]. As shown in Figure 3, TLR2 is significantly $(p<0.05)$ upregulated in response to kidney ligation, but the treatment with high-dose $C$. cicadae attenuated the up-regulation. TLR2 is overexpressed on the surface of the macrophage and recognizes conserved pathogen-associated motifs present on diverse bacteria, fungi, parasites, and viruses. When activated, TLR2 activates the downstream adapter protein MyD88 and ultimately drives the activation of the pro-inflammatory transcription factor NF-KB, thus leading to the upregulation of pro-inflammatory cytokines, i.e., TNF- $\alpha$, IL-1 $\beta$, and IL-6 [20-22]. TLR2 and NF-KB pathway is activated in UUO-operated mice which in turn promotes inflammatory response and oxidative stress.

The inhibitory properties of $C$. cicadae on TLR2 and NF-KB pathway might be related to the 
regulation of pro-inflammatory factors release and activity of antioxidant enzymes, which are believed to be closely related to fibrosis.Natural compounds with significant inhibitory potential of NF-KB pathway can serve as an effective therapeutic candidate for inflammatory diseases $[23,24]$. According to the obtained data from the current study, the $C$. cicadae fruiting have an effective role against inflammatory diseases. However, additional in vivo studies on the mechanism of $C$. cicadae against inflammation is needed for further clinical studies.

\section{CONCLUSION}

The findings of this study demonstrate that $C$. cicadae treatment can protect against the development of UUO-induced tubulointerstitial fibrosis via a mechanism that may be associated with altered renal macrophage polarization and suppression of aberrant TLR2/MyD88/NF-kB pathway signaling. These data thus suggest that C. cicadae preparations may be useful for the treatment of RIF, but further research is required for the validation of these findings.

\section{DECLARATIONS}

\section{Acknowledgement}

This study was funded by National Science Foundation of China (Nos. 81873245); Municipal Human Resources Development Program for Outstanding Leaders in Medical Disciplines in Shanghai (no. 2017BR023); "Shuguang Program" supported by Shanghai Education Development Foundation (16SG37); Senior Talents Program of Integrated Traditional Chinese and Western Medicine in Shanghai (no. ZY(2018-2020)-RCPY-2002)

\section{Conflict of interest}

No conflict of interest is associated with this work.

\section{Contribution of authors}

We declare that this work was done by the authors named in this article and all liabilities pertaining to claims relating to the content of this article will be borne by the authors. Yifei Zhong and Rong Zhu designed all the experiments and revised the manuscript. Rong Zheng and Yuhuang Wang performed the experiments and wrote the manuscript.

\section{Open Access}

This is an Open Access article that uses a funding model which does not charge readers or their institutions for access and distributed under the terms of the Creative Commons Attribution License (http://creativecommons.org/licenses/by/ 4.0) and the Budapest Open Access Initiative (http://www.budapestopenaccessinitiative.org/rea d), which permit unrestricted use, distribution, and reproduction in any medium, provided the original work is properly credited.

\section{REFERENCES}

1. Paterson RR. Cordyceps: a traditional Chinese medicine and another fungal therapeutic biofactory? Phytochemistry 2008; 69: 1469-1495.

2. Kuo YC, Weng SC, Chou CJ, Chang TT, Tsai WJ. Activation and proliferation signals in primary human $T$ lymphocytes inhibited by ergosterol peroxide isolated from Cordyceps cicadae. Br J Pharmacol 2003; 140: 895-906.

3. Jin Z, Chen Y. The Mechanism Study of Cordyceps Sobolifera Mycelium Preventing the Progression of Glomerulosclerosis. Chin J Integr Tradit Western Nephrol 2005; 6: 132-136.

4. Jin Z, Chen Y, Yueyi D. Clinical Observation on Cordyceps Cicadae Shing Tang in Preventing the Progression of Chronic Renal Failure. Chin Archives Tradit Chin Med 2006; 24: 1457-1459.

5. Yang NN, Jiang N, Ma QY, Kong FD, Xie QY, Zhou LM, Yu ZF, Zhao YX. Chemical study of the strain Cordyceps spp. from cell fusion between Cordyceps militaris and Cordyceps cicadae. J Asian Nat Prod Res 2018; 1 -7.

6. Bruck K, Stel VS, Gambaro G, Hallan S, Volzke H, Arnlov J, Kastarinen M, Guessous I, Vinhas J, Stengel B, et al. CKD Prevalence Varies across the European General Population. J Am Soc Nephrol 2016; 27: 2135-2147.

7. Webster AC, Nagler EV, Morton RL, Masson P. Chronic Kidney Disease. Lancet 2017; 389: 1238-1252.

8. Zhong Y, Deng Y, Chen Y, Chuang PY, Cijiang He J. Therapeutic use of traditional Chinese herbal medications for chronic kidney diseases. Kidney Int 2013; 84: 1108-1118.

9. Zheng R, Zhu R, Li X, Li X, Shen L, Chen Y, Zhong Y, Deng Y. N6-(2-Hydroxyethyl) Adenosine From Cordyceps cicadae Ameliorates Renal Interstitial Fibrosis and Prevents Inflammation via TGFbeta1/Smad and NF-kappaB Signaling Pathway. Front Physiol 2018; 9: 1229.

10. World Health Organization. Principles of laboratory animal care. WHO Chron 1985; 39: 51-56

11. Fujimoto S, Satoh M, Horike $H$, Hatta $H$, Haruna $Y$, Kobayashi S, Namikoshi T, Arakawa S, Tomita N, Kashihara N. Olmesartan ameliorates progressive glomerular injury in subtotal nephrectomized rats

Trop J Pharm Res, October 2020; 19(10): 2158 
through suppression of superoxide production. Hypertens Res 2008; 31: 305-313.

12. Modlinger PS, Wilcox CS, Aslam S. Nitric oxide, oxidative stress, and progression of chronic renal failure. Semin Nephrol 2004; 24: 354-365.

13. Zhu R, Chen Y, Deng Y, Wang L. The Mechanism Study of Artificial Culture Cordyceps Sobolifera Mycelium Preventing the Progression of Glomerulosclerosis. Chin J Integr Tradit Western Nephrol 2005; 6: 70-74.

14. Lee S, Huen S, Nishio H, Nishio S, Lee HK, Choi BS, Ruhrberg C, Cantley LG. Distinct macrophage phenotypes contribute to kidney injury and repair. J Am Soc Nephrol 2011; 22: 317-326.

15. Ko GJ, Boo CS, Jo SK, Cho WY, Kim HK. Macrophages contribute to the development of renal fibrosis following ischaemia/reperfusion-induced acute kidney injury. Nephrol Dial Transplant 2008; 23: 842-852.

16. Peng X, Xiao Z, Zhang J, Li Y, Dong Y, Du J. IL-17A produced by both gammadelta $T$ and Th17 cells promotes renal fibrosis via RANTES-mediated leukocyte infiltration after renal obstruction. J Pathol 2015; 235 : 79-89.

17. Huuskes BM, Wise AF, Cox AJ, Lim EX, Payne NL, Kelly $D J$, Samuel CS, Ricardo SD. Combination therapy of mesenchymal stem cells and serelaxin effectively attenuates renal fibrosis in obstructive nephropathy. FASEB J 2015; 29: 540-553.
18. Engel DR, Krause TA, Snelgrove SL, Thiebes S, Hickey MJ, Boor P, Kitching AR, Kurts C. CX3CR1 reduces kidney fibrosis by inhibiting local proliferation of profibrotic macrophages. J Immunol 2015; 194: 16281638.

19. Li F, Xiang J. Signaling pathways regulating innate immune responses in shrimp. Fish Shellfish Immunol 2013; 34: 973-980.

20. Kawai T, Akira S. The role of pattern-recognition receptors in innate immunity: update on Toll-like receptors. Nat Immunol 2010; 11: 373-384.

21. Taro K, Shizuo A. Toll-like receptors and their crosstalk with other innate receptors in infection and immunity. Immunity 2011; 34: 637-650.

22. Akira S, Uematsu S, Takeuchi O. Pathogen recognition and innate immunity. Cell 2006; 124: 783-801.

23. Yamamoto $Y$, Gaynor RB. Therapeutic potential of inhibition of the NF-kappaB pathway in the treatment of inflammation and cancer. J Clin Invest 2001; 107: 135142.

24. Lu MY, Chen CC, Lee LY, Lin TW, Kuo CF. N(6)-(2Hydroxyethyl)adenosine in the Medicinal Mushroom Cordyceps cicadae Attenuates LipopolysaccharideStimulated Pro-inflammatory Responses by Suppressing TLR4-Mediated NF-kappaB Signaling Pathways. J Nat Prod 2015; 78: 2452-2460. 